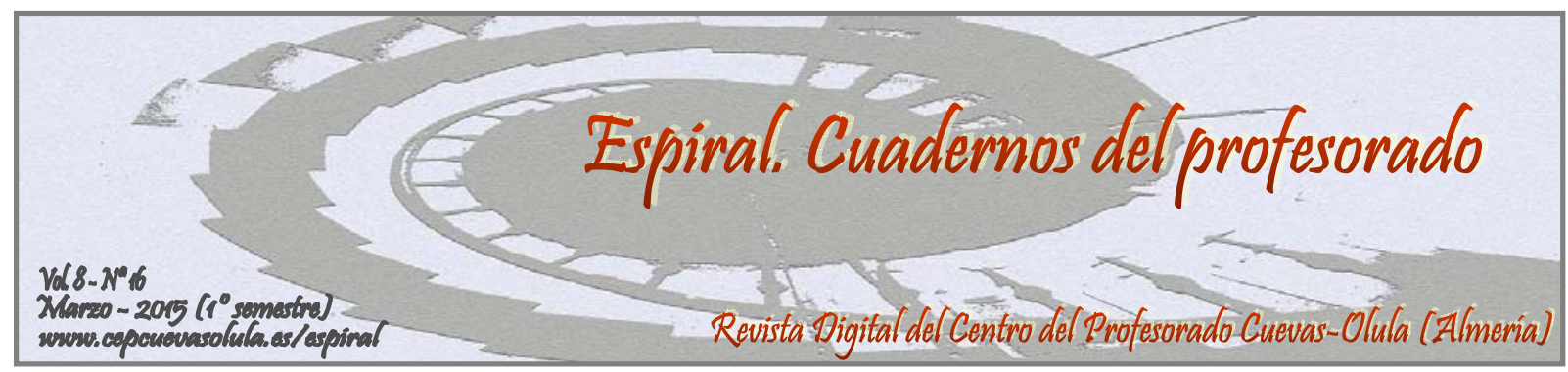

\title{
EVOLUCIÓN DE LAS INVESTIGACIONES SOBRE LA ENSEÑANZA DE LAS ACTIVIDADES EN EL MEDIO NATURAL EN EDUCACIÓN FÍSICA ESCOLAR
}

\author{
PUBLICATIONS ABOUT PHYSICAL ACTIVITIES IN THE NATURAL \\ ENVIRONMENT OF THE SPANISH PHYSICAL EDUCATION'S JOURNALS
}

\author{
Antonio Baena-Extremera, Patricio Palao Puche \\ y Daniel Jara Cortina \\ Facultad de Ciencias del Deporte, Universidad de Murcia, España
}

\begin{abstract}
RESUMEN: El presente trabajo consiste en la concreción del volumen de publicaciones relacionadas con las Actividades Físicas en el Medio Natural por las revistas españolas desde el año 1992 hasta el 2013. Para ello se realizó una revisión documental descriptiva en 30 revistas españolas ingresadas en la plataforma DICE y relacionadas con la Educación Física y el Deporte. Tras la realización de la revisión documental, se introdujeron los resultados obtenidos en una base de datos y a raíz de esta se empezó a resolver el objeto de estudio. Los resultados muestran que el total de artículos publicados en este período de tiempo entre todas las revistas investigadas es de 267 artículos. Y que las revistas españolas publican cada vez más sobre las Actividades Físicas en el Medio Natural. Esto pone de manifiesto que estos contenidos están en auge en la investigación dentro de la ciencia de la Actividad Física y el Deporte.
\end{abstract}

Palabras clave: deporte, aventura, naturaleza, revisión.

ABSTRACT: The aim of this article is to provide a precise overview of the publications in Spanish journals concerned with physical activities in the natural environment between 1992 and 2013. For this purpose, a descriptive revision of articles from 30 Spanish journals accessed on the virtual platform DICE was made. Once the revision was completed, a total number of 267 articles had been analyzed, which results were introduced into a database utilized to accomplish this study. The conclusions show that the number of publications in Spanish journals about physical activities in natural environment is increasing. This fact evinces the rising popularity of the research on this topic in the field of Physical Activity and Sports.

Key words: sport, adventure, nature, review.

Baena-Extremera, A., Palao Puche, P., y Jara Cortina, D. (2015). Evolución de las investigaciones sobre la enseñanza de las actividades en el medio natural en Educación Física escolar. Espiral. Cuadernos del Profesorado, 8(16), 13-18. Disponible en: $\underline{\text { http://www.cepcuevasolula.es/espiral. }}$

Fecha de recepción: 30/09/2014

Fecha de aceptación: 25/01/2015
Enviar correspondencia a: abaenaextrem@um.es 


\section{1.- INTRODUCCIÓN}

La Ciencia de la Actividad Física y el Deporte es una ciencia relativamente joven. En esta ciencia, el perfil profesional establecido por el Libro Blanco (ANECA, 2005), son Educación, Recreación, Salud, Gestión y Alto Rendimiento. Dentro de esta joven pero gran ciencia, nos encontramos con los contenidos relacionados con las Actividades Físicas en el Medio Natural (AFMN).

Se entiende por AFMN, "el estudio y desarrollo de todos aquellos movimientos y técnicas necesarias para la utilización del medio natural según los principios pedagógicos” (Granero, 2007), por lo cual, según esta definición y la clasificación establecida por el Libro Blanco, podemos encajar las AFMN dentro de las diferentes ramas de la Educación Física. Esto ha provocado que la AFMN sea un tema de investigación y de desarrollo en crecimiento dentro de la Ciencia de la Actividad Física y el Deporte, especialmente desde el año 1990, año en el cual se aprobó la Ley Orgánica General del Sistema Educativo (LOGSE). Con esta ley educativa, se implantaron las AFMN como uno de los bloques de contenidos de la asignatura de Educación Física, otorgándoles un valor reconocido a este tipo de prácticas en la formación del alumnado, y asentándose de forma definitiva dentro del currículum en la Educación Física (Peñarrubia, Guillen, y Lapetra, 2013). Las AFMN incluidas dentro del currículum, tienen muchas formas de expresión y a través de ellas es posible educar en temas transversales, como el tratamiento de la educación ambiental, temas ya desarrollados con anterioridad por Villalvilla (2001), Mediavilla (2003), Tejedor (2003), Gómez-Limón (2008) y Peñarrubia (2008).

Además de la implantación de la citada ley educativa, los I.N.E.F.s incluyeron dentro de sus planes de estudios, una asignatura con el mismo nombre, dentro de la formación de los licenciados en Educación Física. Es a partir de estos hechos, cuando las publicaciones entorno a las AFMN cobran una mayor importancia debido a su carácter educativo y la necesidad de innovación para el desarrollo de estos contenidos dentro de las clases de Educación Física. Más adelante, se empezaría a investigar en nuestro país sobre otros contenidos de medio natural, relacionados con el rendimiento, la gestión y la recreación. De esta forma, empezaron a surgir diversos artículos en las revistas españolas, algunos con carácter más de investigación-experimental, otros más de aplicaciones prácticas para profesores, otros más de revisión, etc.

Las revistas científicas son la forma de información y foro entre expertos académicos sobre una materia (Martínez, Reig y Marín, 2013). Para poder progresar en el estudio de esta materia es necesario conocer lo escrito hasta el momento para poder tomar ideas de ello y renovar los conocimientos adquiridos y aumentar el nivel de dominio del tema. Para poder hacernos una idea sobre el gran elenco de artículos publicados sobre AFMN en nuestro país, vamos a seguir una clasificación determinada, según dos parámetros: 1. Tipología dentro de la cual entendemos que existen artículos de investigación, revisión bibliográfica, de reflexión y de aplicación didáctica (Tabla 1). 2. Contenido en el cual consideramos que hay diferentes artículos con contenido teórico, de deporte, de actividad física, de medio natural y de educación física (Tabla 2).

Tabla1. Según la tipología del artículo.

\begin{tabular}{ll}
\hline Tipo de artículo & \multicolumn{1}{c}{ Ejemplo de artículo en revista española } \\
\hline Investigación & $\begin{array}{l}\text { Análisis del perfil competitivo de los practicantes de deportes de aventura } \\
\text { que compiten a nivel estatal. Baena (2007) }\end{array}$ \\
Revisión bibliográfica & $\begin{array}{l}\text { Factores determinantes del rendimiento en vela deportiva: revisión de la } \\
\text { literatura. Manzanares, Segado y Menayo (2012) }\end{array}$ \\
Reflexión & $\begin{array}{l}\text { Las clases de E.F. como motor de cambio social: reflexionando sobre } \\
\text { actividades en la naturaleza, currículum oculto y valores. Venero (2007) }\end{array}$ \\
Aplicación didáctica & $\begin{array}{l}\text { Deportes de aventura “indoor”: enseñanza de la espeleología en los } \\
\text { institutos de educación secundaria. Baena y Granero (2009) }\end{array}$ \\
\hline
\end{tabular}


Tabla 2. Según el Contenido del artículo.

\begin{tabular}{|c|c|}
\hline Tipo de artículo & Ejemplo de artículo en revista española \\
\hline Teórico & $\begin{array}{l}\text { Evolución de las AFMN con fines educativos. Pérez, Caballero y Jiménez } \\
\text { (2009) }\end{array}$ \\
\hline Deporte & $\begin{array}{l}\text { Análisis cualitativo de las implicaciones musculares de la escalada deportiva de } \\
\text { alto nivel en competición. Benito, Sedano, Redondo y Cuadrado (2013) }\end{array}$ \\
\hline Actividad Física & $\begin{array}{l}\text { Hacia un modelo de actividades fisicodeportivas inclusivas en el medio natural. } \\
\text { Torrebadella (2013) }\end{array}$ \\
\hline Educación Física & $\begin{array}{l}\text { Aula en bici: un proyecto longitudinal de intervención docente en Ed. Primaria. } \\
\text { Castellar, Pradas, Rapún, Coll y Pérez (2013) }\end{array}$ \\
\hline Medio Natural & $\begin{array}{l}\text { Incorporación de contenidos de AMN mediante la investigación-acción } \\
\text { colaborativa. Guillén y Peñarrubia (2013) }\end{array}$ \\
\hline
\end{tabular}

Teniendo en cuenta lo anterior, el objetivo de este artículo es concretar el volumen de publicaciones relacionadas con la AFMN por las revistas españolas desde el año 1992 hasta el 2013.

\section{2.- MÉTODO}

\section{Diseño}

El diseño llevado a cabo para esta investigación ha sido la revisión documental descriptiva (Sampieri, Collado y Lucio, 1998), de la bibliografía científica más importante a nivel nacional relacionado con las AFMN, para desarrollar un análisis descriptivo en función de los objetivos establecidos. Además, este trabajo tiene un corte longitudinal en su estudio, pues se tienen en cuenta lo publicado a lo largo de todos los años citados.

\section{Instrumentos, fiabilidad documental y procedimiento}

El trabajo de campo se llevó a cabo entre los meses de noviembre a diciembre del año 2013. Por ello, los artículos publicados en 2014, no estarían recogidos en estos resultados.

En España hay muchas revistas relacionadas con la Educación Física que pueden incluir artículos relacionados con la AFMN. Para la elección de las revistas a analizar, hemos utilizados diversos criterios para evaluar la calidad de las mismas, tomando como guía algunas plataformas que evalúan la calidad y la repercusión de las revistas científicas que hay en nuestro país, como pueden ser DICE (Difusión y Calidad Editorial de las revistas Españolas de Humanidades y Ciencias Sociales y Jurídicas), IN-RECS, RESH (Revistas Españolas de Ciencias Sociales y Humanidades)...

Según (Tur et al., 2014), "la especialización de las revistas se desprende de la clasificación que reciben en bases de datos y de los términos utilizados en su información pública" "también indica el grado de consolidación del campo científico", por lo cual si nos basamos en estas plataformas y bases de datos obtendremos fiabilidad en las revistas analizadas.

Para realizar este trabajo, hemos seleccionado concretamente las revistas indexadas e la plataforma DICE en la cual se clasifican las revistas españolas de Ciencias Sociales y Jurídicas según la difusión y la calidad editorial de estas. Dentro de esta plataforma, de entre todas las revistas de investigación encontradas se han elegido 30, que son las que corresponden a Educación Física o afines, alguna de ellas como la "Revista de Psicología del Deporte" con 0.827 de impacto o la revista “Apunts” con 0.293.

Además para llevar a cabo la revisión documental de esta investigación, se han utilizado algunos de los buscadores más importantes en investigación a nivel nacional, como CSIC (ICYT, IME, ISOC), DIALNET, entre otras. Igualmente, se han utilizado bases de datos internacionales, como ISI Web of Knowledge, Scopus y Sports Discus.

Para ello, los descriptores introducidos para realizar dicha búsqueda, han sido: 
- Actividades en la naturaleza.

- Actividades físicas en el medio natural.

- Aventura.

Además de estos buscadores, se ha realizado un análisis revista por revista, desde su página web correspondiente, revisando todos y cada uno de los números desde el año 1992 hasta el año 2013. Por todo esto, la fiabilidad documental podemos asegurar que es alta, aunque hay que destacar, que la subjetividad de los evaluadores, es un sesgo a tener en cuenta a la hora de decidir si un artículo está relacionado o no con las AFMN o si pertenece a un tipo de artículo u otro.

\section{3.- RESULTADOS}

\section{Volúmenes de publicaciones desde el año 1992 hasta el año 2013}

Tras la revisión de la literatura en 30 revistas españolas específicas de Ciencias de la Actividad Física y el deporte desde el año 1992 hasta el año 2013, hemos obtenido un total de 268 publicaciones en forma de artículo. Este número de artículos los hemos encontrado en un total de 26 revistas, ya que de las 30 originales con las que se empezó la revisión, en cuatro de ellas no se ha hallado en ninguno de sus artículos un contenido relacionado con las AFMN, deportes de aventura, etc. Las cuatro revistas descartadas por insuficiencia de resultados relacionados con el objeto de estudio fueron las siguientes:

- Coaching \& Sport Science Review

- FEGUI. Revista en Salvamento Acuático y Primeros Auxilios

- Movimiento Humano

- Revista Internacional de Deportes Colectivos

Los resultados obtenidos en relación al volumen de publicaciones y los años de publicación, los encontramos en la siguiente gráfica.

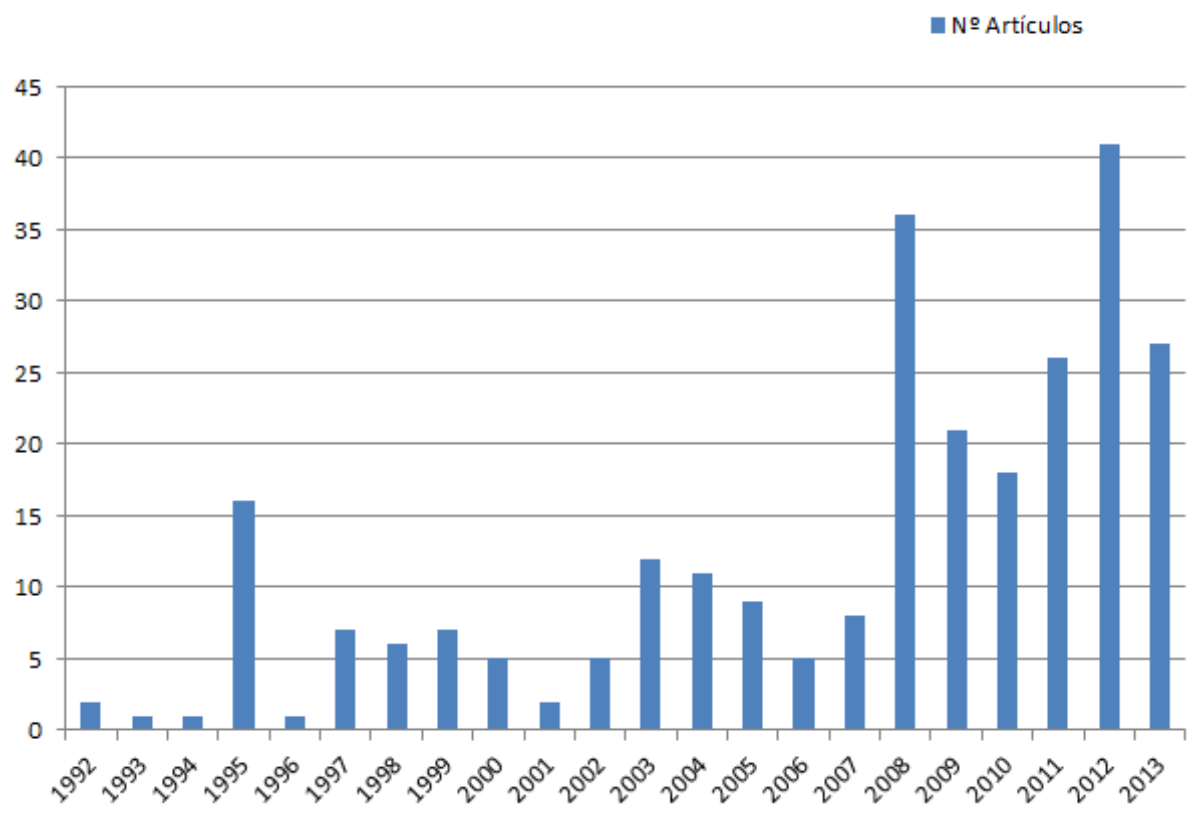

Figura 1. Volumenes de publicaciones entre 1992 y 2013.

En relación a la gráfica observamos como la tendencia en los últimos años ha sido al aumento del número de publicaciones. En la década de los años 90 encontramos un pico, concretamente en el año 1995 el cual se debe a que la revista “Apunts” en su número 41, la cual realizó un monográfico 
sobre las AFMN. Esto supuso un gran aumento de las publicaciones en este año con 15 artículos solo en su tomo número 41. En ese año fue la única revista que publicó sobre el tema con un $6 \%$ del total de las publicaciones de todo el periodo de tiempo. De esta forma, se obtuvieron un total de 16 artículos en el año 1995, 15 artículos en la revista número 41 y un artículo en la número 42.

En la primera década del siglo XXI apreciamos que el punto de mayor volumen de artículos relacionados lo encontramos en el año 2008. En este año el total de las publicaciones, se reparte en 10 revistas diferentes. Con un total de 36 artículos publicados, cabe destacar en este año la revista "Wanceulen" con un monográfico sobre AFMN que incluye 13 publicaciones de diversa índole. En este mismo año la revista Ágora en su número 7 y 8 contempla otro monográfico llamado "El aula naturaleza” en el que se recogen un total de 7 artículos.

Cabe destacar también aunque no se contempla como uno de los picos sobresalientes en la gráfica, que en el año 2011 la revista "Journal of Human Sport and Exercice” publicó un monográfico específico sobre triatlón (deporte estrechamente relacionado con el medio natural), el cual supone más del $50 \%$ de las publicaciones totales recogidas en este año.

El pico máximo en todos los años registrados lo encontramos en el año 2012 con un total de 41 artículos editados. De nuevo volvemos a encontrar un monográfico en la revista digital "EmásF". Pero lo positivo del aumento de estas publicaciones, es que aunque encontremos de nuevo un monográfico observamos que el total de las revistas que publican este año son 13 y en el año 2013 el total de revistas han sido 10 con un total de 27 artículos, sin ningún monográfico.

\section{4.- CONCLUSIONES}

Para concluir, consideramos que este estudio nos sirve de guía para conocer que las AFMN son un tema al alza y que cada vez se investiga más sobre estos contenidos, siendo diferentes las revistas que se implican más en este tema, publicando algún artículo en concordancia. Por ello era necesario un estudio como este, para conocer de dónde venimos y hacia dónde vamos, sabiendo donde se concentra la mayor parte de la literatura en las revistas españolas y en que años hay más volumen de publicaciones, lo que sirve para los estudiosos interesados en esta materia como referente para la búsqueda de información.

Siempre teniendo en cuenta que la realización de este estudio se llevo a cabo tomando como aceptados dentro de la materia de AFMN los artículos que consideraban los autores de este artículo, y por lo cual pueda ocasionar alguna discrepancia con otros autores que puedan interpretar de otra manera la literatura analizada.

Este artículo es el inicio de futuros trabajos donde se profundizará sobre este estudio donde se analizarán los volúmenes de publicaciones desde diferentes perspectivas, para que el conjunto completo del estudio sea la guía perfecta de referencia para los investigadores españoles e internacionales sobre la AFMN en relación a la revisión documental descriptiva realizada.

\section{5.- REFERENCIAS}

Baena, A. (2007). Análisis del perfil competitivo de los practicantes de deportes de aventura que compiten a nivel estatal. Red: Revistas de entrenamiento deportivo, 21 (3), 25-32.

Baena, A. y Granero, A. (2009). Deportes de aventura “indoor”: enseñanza de la espeleología en los institutos de educación secundaria. Tándem: Didáctica de la educación física, 30, 47-60.

Castellar, C., Pradas, F., Rapún, M., Coll, I., y Pérez, S, (2013). Aula en bici: un proyecto longitudinal de intervención docente en Ed. Primaria. Retos, 23, 5-9.

De Benito, A., Sedano, S., Redondo, C., y Cuadrado, G. (2013). Análisis cualitativo de las implicaciones musculares de la escalada deportiva de alto nivel en competición. RICYDE. Revista Internacional de Ciencias del Deporte, 9(32), 154-180.

Gómez-Limón, J. (2008). Ocio y deporte en espacios naturales protegidos: Planificación, dirección e impactos ambientales asociados. En Miguel, A. y López, J.J. (Coords.), Cuadernos Técnicos 13, La Formación en la profesión de Educación Física escolar (pp. 101-122). Palencia: Patronato Municipal de Deportes. 
Granero, A. (2007). Una aproximación conceptual y taxonómica a las actividades físicas en el medio natural. Lecturas: Educación Física y Deportes. http://www.efdeportes.com/ (107).

Guillén, A. y Peñarrubia, C. (2013). Incorporación de contenidos de actividad en el medio natural mediante la investigación-acción colaborativa. Retos, 23, 23-28.

Libro Blanco Ciencias de la Actividad Física y el Deporte. Agencia Nacional de Evaluación de la Calidad. [Consultado el 20/01/2014] http://www.aneca.es/var/media/150296/libroblanco_deporte_def.pdf

Manzanares, A. Segado, F y Menayo, R. (2012). Factores determinantes del rendimiento en vela deportiva: revisión de la literatura. Cultura, Ciencia y Deporte. 20, 125-134.

Martínez, R., Reig, R., y Marín, M. (2013). Una década del mensaje sobre la salud en las revistas de comunicación indexadas en IN-RECS (2000-2011). Estudios sobre el mensaje periodístico, 19, 10471059.

Mediavilla, L. (2003). Análisis del impacto ambiental de las actividades físicas en el medio natural. En Miguel, A. (Comp.), Actas del I Congreso Internacional Actividades Físicas en el Medio Natural en la Educación Física escolar (pp. 222-229). Palencia: Escuela Universitaria de Educación.

Peñarrubia, C. (2008). Actividades y propuestas para fomentar la educación ambiental en un campamento juvenil. IV Congreso Internacional de Educación Ambiental (paper). Madrid: Facultad de Educación de la Universidad Complutense.

Peñarrubia, C., Guillen, R., y Lapetra, S. (2013). Evolución de las actividades en el medio natural en educación física a partir de 1990. Ágora, 2(15), 113-129.

Pérez, R. Caballero, J., y Jiménez, L. (2009). Evolución de las actividades físicas en el medio natural con fines educativos. Materiales para la historia del deporte, 7, 59-76.

Real Decreto 1631/2006, 29 diciembre, que establece las enseñanzas mínimas en la ESO. BOE (106).

Sampieri, R. H., Collado, C. F., y Lucio, P. B. (1998). Metodología de la investigación. Segunda edición. México: Mcgraw-Hill Interamericana Editores.

Tejedor, J. C. (2003). Aplicación curricular de las actividades en el medio natural a través de la Educación Física escolar. En Miguel, A. (Comp.), Actas del I Congreso Internacional Actividades Físicas en el Medio Natural en la Educación Física escolar. Palencia: Escuela Universitaria de Educación.

Torrebadella, X (2013). Hacia un modelo de actividades físico-deportivas inclusivas en el medio natural. EmásF: revista digital de educación física, 23, 25-39.

Tur, V., López, M. C., García, J. A., López, M., Monserrat, J., y Quiles, M. C., (2014). Especialización y revistas académicas españolas de comunicación. Revista Latina de Comunicación Social, 69, 12-40.

Venero, J. P. (2007). Las clases de Educación Física como motor de cambio social: reflexionando sobre actividades en la naturaleza, currículum oculto y valores. Retos, 11, 51-53.

Villalvilla, H. (2001). Deporte y naturaleza. El impacto de las actividades deportivas y de ocio en el medio natural. Madrid: Talasa

Villar, F. (2005). Libro Blanco Título de Grado en Ciencias de la Actividad Física y el Deporte. ANECA. 